\title{
Cytoplasmic String Between ICM and mTE Is a Positive Predictor of Clinical Pregnancy and Live Birth Outcomes in Elective Frozen-thawed Single Blastocyst Transfer Cycles: a Time-lapse Study
}

\section{Bing-Xin Ma}

Tongji Hospital of Tongji Medical College of Huazhong University of Science and Technology

\section{Lei Jin}

Tongji Hospital of Tongji Medical College of Huazhong University of Science and Technology Bo Huang ( $\sim$ hb@tjh.tjmu.edu.cn )

Reproductive Medicine Center, Tongji Hospital, Tongji Medical College, Huazhong University of Science and Technology https://orcid.org/0000-0001-8897-3306

\section{Research}

Keywords: cytoplasmic string, elective single blastocyst transfer, inner cell mass, trophectoderm, time lapse

Posted Date: December 11th, 2020

DOI: https://doi.org/10.21203/rs.3.rs-122470/v1

License: (9) This work is licensed under a Creative Commons Attribution 4.0 International License.

Read Full License 


\section{Abstract}

Background: In this study, we aim to investigate whether cytoplasmic string between inner cell mass (ICM) and mural trophectoderm ( $\mathrm{MTE}$ ) is a positive predictor of clinical pregnancy and live birth outcomes.

Methods: 1,267 elective frozen-thawed single blastocyst transfer (eSBT) cycles cultured in time-lapse incubation system from January 2018 to May 2019 were involved in the study. Blastocysts were grouped according to the appearance of cytoplasmic strings between ICM and mTE cells, and identified as "Present" and "Absent" groups. In Present group, they were further categorized according to the quantity of cytoplasmic strings between ICM and mTE cells. Clinical pregnancy and live birth outcomes of blastocysts were used to evaluate the effect of cytoplasmic strings between ICM and mTE.

Results: The baseline demographic and laboratory features were similar between the Present and Absent groups of cytoplasmic strings between ICM and mTE ( $P>0.05)$. According to the time-lapse analysis, cytoplasmic strings between ICM and mTE were more visible among good quality blastocysts.

Furthermore, blastocysts with cytoplasmic strings showed a higher clinical pregnancy and live birth rates $(P<0.05)$, and no significant differences were observed in abortion rate and birth weight $(P>0.05)$.

Conclusions: Although the previous conclusions of cytoplasmic strings were controversial, the present time-lapse analysis provides the evidence for the first time that cytoplasmic strings between ICM and mTE cells would be a positive predictor of clinical pregnancy and live birth outcomes in elective frozenthawed single blastocyst transfer cycles.

\section{Background}

The goal of assist reproductive technology is to achieve a healthy singleton birth and minimize maternal and neonatal risk [1, 2]. In order to realize the goal, elective single blastocyst transfer (eSBT) was preferred during these decades[3, 4]. Blastocyst transfer is considered as a more physiologically appropriate time due to the more closely mimics the time of natural implantation and may improve synchrony between the endometrium and embryo development $[5,6]$. It is reported that the clinical pregnancy and live birth rates of elective single blastocyst transfer (eSBT) are similar with double embryos transfer, and higher than those with single cleavage-stage embryo transfer. Furthermore, eSBT generates a reduced incidence of multiple pregnancy than double embryos transfer [7-9].

With the development of cryopreservation technology, more embryos were selected to be cryopreserved for further use. It is reported that frozen-thawed embryos transfer could lead to a higher live birth rate in women with polycystic ovary syndrome. Besides, frozen-thawed embryos transfer could avoid the exposure of the endometrium to the adverse sequelae of ovarian stimulation[10]. Besides, supraphysiologic hormonal milieu increases the incidence of ectopic pregnancy in fresh cycles. So, frozen-thawed eSBT strategy could not only optimize pregnancy rates, but also maintain perinatal safety[11].Blastocyst selection is the key to a successful eSBT strategy, as it is important to shorten the 
time to get pregnancy [12]. In the past, morphological assessment was the main criterion to select blastocysts. The blastocyst grading system by Gardner and Schoolcraft remains largely unchallenged and widely used in clinic [13]. However, this system did not include some important morphological parameters often observed in the in vitro fertilization (IVF) laboratory. Albeit of unknown importance, some of these parameters were briefly introduced in the Istanbul consensus document, i.e. the formation of cytoplasmic strings often linking together different cells and cell types [14].

Methods for embryo selection have greatly improved over the past decade, not only static morphological assessment, but also integration of no-invasive time-lapse analysis. Cytoplasmic strings could be observed between oocyte and zona pellucida (ZP), the outside surface of blastomere and traversing from inner cell mass (ICM) and mural trophectoderm (mTE) by time-lapse system. It is reported that these strings would be required to make possible a selective and bidirectional communication between mitotic mTE cells and ICM cells [15]. But, the impact of cytoplasmic strings on the clinical outcome of blastocyst is controversial [16].

In this study, we aim to determine whether the cytoplasmic strings between ICM and mTE cells is a positive predictor of clinical pregnancy and live birth in frozen-thawed eSBT cycles.

\section{Methods}

\section{Study design}

In the present study, we retrospectively analyzed 3,223 transfer cycles with a time-lapse incubation system (EmbryoScope Plus, Vitrolife, Sweden) from January 2018 to May 2019 at Reproductive Medicine Center, Tongji Hospital, Tongji Medical College, Huazhong University of Science and Technology. 1,537 fresh cycles and 1686 frozen embryo transfer (FET) cycles were involved (Fig. 1). Among FET cycles, 194 cleavage embryos and 1,492 blastocysts were transferred. The eSBT cycles were 1,330 among all blastocysts transfer. After exclusion of no-known implantation data (KID) eSBT cycles and loss of followup, 1,267 eSBT cycles were involved in the study eventually. The study conformed to the Declaration of Helsinki for Medical Research involving Human Subjects. It was approved by the Ethical Committee of Reproductive Medicine Center, Tongji Hospital, Tongji Medicine College, Huazhong University of Science and Technology.

\section{Clinical Protocol}

According to the characteristics of patients, main controlled ovarian stimulation (COS) were performed as per our previous paper $[17,18]$. During COS, patients were monitored closely using transvaginal ultrasound. Human chorionic gonadotropin (HCG) was given when the leading follicle(s) was $>18 \mathrm{~mm}$. Thirty-six hours after HCG injection, ultrasound-guided oocytes retrieval was carried on.

\section{Laboratory Protocol}


The protocol of semen/cumulus-oocyte complexes (COCs) preparation, insemination and embryo culture were performed as our previous paper [19]. Density gradient centrifugation was used to optimize the semen samples. The concentration, motility and morphology of sperm were evaluated according to the fifth edition of WHO guidelines. In IVF cycles, each COC was inseminated with 10,000 motile spermatozoa 3-4 $\mathrm{h}$ after retrieval. In intracytoplasmic sperm injection (ICSI) cycles, the COCs were denuded 2 hours later, and sperm was injected 4 hours later after retrieval. Then, zygotes were transferred to G1 Plus (Vitrolife, Sweden) and cultured in time-lapse incubation system. The system was set to take pictures for each embryo every $10 \mathrm{~min}$. Pronuclei were checked 16-18 h later after insemination. On Day 3 , the media was replaced by G2 Plus (Vitrolife, Sweden). On Day 5 and 6, blastocysts were cryopreserved for further use. All culture media were Vitrolife G-series media (Vitrolife, Sweden).

\section{FET protocols}

The FET protocols were referred to our previous papers. For natural cycles, the endometrial thickness, follicle growth, and ovulation were measured by transvaginal ultrasound examination and measurement of the serum progesterone levels were initiated from cycle days 10 to 12. Thawing and transferring of blastocysts was planned 3 days after ovulation. From 1 day after ovulation, progesterone is injected intramuscularly for luteal support.

For hormone replacement treatment cycles, oral estradiol (Progynova; Bayer; Leverkusen) signed $2 \mathrm{mg} /$ day from 1 to 4 cycle days, $4 \mathrm{mg} /$ day from days 5 to 8 days, and $6 \mathrm{mg} /$ day from 9 to 12 days. On the 13th day, when the thickness of the endometrium reaches $8.0 \mathrm{~mm}$ or the maximum, $40 \mathrm{mg}$ of progesterone is injected intramuscularly for 3 days. The blastocyst was thawed and transplanted on the 4th day after 3 days of progesterone administration.

Four weeks after transfer, the fetal cardiac activity in the uterus was observed transvaginal ultrasound to determine the clinical pregnancy. Miscarriage was defined as loss of the gestational sac or fetal heart activity within 20 weeks after confirming clinical pregnancy.

\section{Grouping}

Blastocysts were grouped according to the appearance of cytoplasmic strings between ICM and mTE cells, and identified as "Present" and "Absent" groups. In Present group, they were further categorized according to the quantity of cytoplasmic strings between ICM and mTE cells as follows: single or double string(s) (Class I); three to four strings (Class II) and five strings or more strings (Class III) (Fig. 2).

\section{Statistical Analysis}

Statistical analysis was performed by the Statistical Package for Social Sciences (SPSS) version 19.0 (SPSS Inc., USA). T-test was used as a continuous variable to compare difference, such as age, Body Mass Index (BMI), duration of subfertility, FSH, AMH, oocytes MII, 2PN, and so on. Chi-square test was used for binary and categorical variables, such as primary subfertility, insemination, clinical pregnancy, and so on. Statistical significance was established at $\mathrm{P}<0.05$. 


\section{Results}

\section{Patient and cycle characteristics}

A total of 1,267 frozen-thawed eSBT cycles at Reproductive Medicine Center, Tongji Hospital, Tongji Medical College, Huazhong University of Science and Technology were involved in the cohort study. According to the appearance of cytoplasmic strings between ICM and mTE cells, they were allocated as "Present" group with 1,002 cycles and "Absent" group with 265 cycles. So, 79.08\% transferred blastocysts could be observed with cytoplasmic strings between ICM and mTE cells, and $20.92 \%$ could not (Fig. 3A).

Patients in the two groups were similar in baseline demographic features. The difference between the average maternal ages, maternal BMI, ratio of primary subfertility, duration of subfertility, the value follicle stimulating hormone $(\mathrm{FSH})$ and anti-mullerian hormone $(\mathrm{AMH})$ of the two groups were not significant $(\mathrm{P}$ $>0.05$, Table 1). Therefore, the baseline demographic features of the two groups were similar. The cycle characteristics, such as insemination method, oocytes, MII, 2PN, FET protocol and the endometrial thickness were all similar $(P>0.05$, Table 1$)$. 
Table 1

Baseline demographic features of the included patients and cycles

\begin{tabular}{|llll|}
\hline & $\begin{array}{l}\text { Present } \\
(\mathbf{n}=1,002)\end{array}$ & $\begin{array}{l}\text { Absent } \\
(\mathbf{n}=265)\end{array}$ & $\mathbf{P}$ \\
\hline Maternal age & $31.18 \pm 4.46$ & $31.42 \pm 4.54$ & $>0.05$ \\
\hline Maternal BMI & $21.72 \pm 3.81$ & $21.94 \pm 3.09$ & $>0.05$ \\
\hline Duration of subfertility & $3.14 \pm 2.40$ & $3.53 \pm 2.38$ & $>0.05$ \\
\hline Primary subfertility & $711 / 1002(70.96)$ & $190 / 265(71.7)$ & $>0.05$ \\
\hline FSH & $7.45 \pm 2.05$ & $7.45 \pm 1.89$ & $>0.05$ \\
\hline AMH & $6.48 \pm 4.25$ & $6.35 \pm 4.09$ & $>0.05$ \\
\hline IVF Fertilization & $582 / 1002(58.08)$ & $158 / 265(59.62)$ & $>0.05$ \\
\hline ICSI Fertilization & $420 / 1002(41.92)$ & $107 / 265(40.38)$ & $>0.05$ \\
\hline Oocytes & $13.46 \pm 3.92$ & $13.69 \pm 3.63$ & $>0.05$ \\
\hline MII & $11.58 \pm 3.29$ & $11.80 \pm 3.26$ & $>0.05$ \\
\hline 2PN & $8.25 \pm 2.86$ & $8.73 \pm 3.07$ & $>0.05$ \\
\hline Hormone replacement treatmnt cycles & $977 / 1002$ & $258 / 265$ & $>0.05$ \\
\hline Natural cycles & $(97.50)$ & $(97.36)$ & $>0.05$ \\
\hline Endometrial thickness & $25 / 1002(2.50)$ & $7 / 265(2.64)$ & $>0.05$ \\
\hline Data are presented as mean \pm SD or number $(\%)$. & $9.46 \pm 1.33$ & \\
\hline & $9.50 \pm 1.52$ & & \\
\hline
\end{tabular}

Among all blastocysts observed with cytoplasmic strings between ICM and mTE cells, namely "Present" group, three classifications were divided according to the quantity of cytoplasmic strings. As shown in Fig. 3B, 572 blastocysts were allocated to Class I, 366 blastocysts to Class II and 64 blastocysts to Class III. The percentages were $57.09 \%, 36.53 \%$ and $6.39 \%$ in Class I, II and III, respectively. So, single and double cytoplasmic strings between ICM and mTE cells were more visible.

\section{The frequency of strings observed in different blastocyst stages}

Most transferred blastocysts that meet the criterion were cultured on Day 5 and Day 6 . Few blastocysts culture was prolonged to Day $7(\mathrm{~N}=3)$. As shown in Fig. 4A, the percentage of the appearance of cytoplasmic strings between ICM and mTE cells were similar from Day 5 to Day $7(P<0.05)$. 
The blastocyst development stage and quality of ICM and TE were was referenced to David Gardner as follow: Stage 2: the volume of the blastocoel is not less than half of the total volume of the blastocyst; Stage 3: the blastocoel completely occupies the total volume of the blastocyst; Stage 4: the blastocyst expands, the blastocoel completely fills the blastocyst, the total volume of the embryo becomes larger, and the zona pellucida becomes thinner; Stage 5: the hatching blastocyst; Stage 6: the blastocyst hatched from ZP [13]. In most cases, the vitrification criterion is larger than Stage 2. So, only 21 cases at Stage 2 were vitrificated. As shown in Fig. 4B, except Stage 2, other stages were present a similar percentage of appearance of cytoplasmic strings between ICM and mTE cells, from $72.7-90.00 \%(P<$ 0.05). Only $28.6 \%$ blastocysts at Stage 2 could be observed with the cytoplasmic strings between ICM and $\mathrm{mTE}$ cells.

The ICM/TE grades are also referenced to David Gardner as follow: A. a large number of cells and closely packed; $B$. a small number of cells and loosely packed; $C$. very few cell numbers [13]. The percentages of stings appearance were significantly less in blastocysts with a Grade $C$ of both ICM and TE than that with Grades A and B (Fig. 4C and D). So, the appearance of strings was rare in low-quality blastocysts.

\section{Pregnancy and perinatal outcomes}

The main outcome of the study was the clinical pregnancy rate and live birth rate. As shown in Fig. 5A, $59.1 \%$ blastocysts were implanted in Present group, and $48.2 \%$ blastocysts were implanted in Absent group. So, appearance of strings may lead to a better clinical pregnancy rate $(P<0.05)$. Among three classifications of blastocysts, Class I and II present a similar clinical pregnancy $(58.8 \%$ and $53.0 \%, \mathrm{P}>$ 0.05), but Class III (97.5\%) is much higher than other groups $(P<0.05)$.

The blastocysts in Present group also achieve a higher live birth rate compared with Absent group (48.5\% versus $35.5 \%, P<0.05$, Fig. $5 B$ ). The results of live birth showed the similar trend with clinical pregnancy rate. The live birth rates of blastocysts in Class I and II were $50.7 \%$ and $40.0 \%$, respectively $(P>0.05)$. But, the rate in Class III was 77.5\%, much higher than the other two groups $(P<0.05)$.

As shown in Fig. 5C, the abortion rate was not significantly different in all groups. Though, the abortion rate is Class III reached $20.0 \%$, the case number was too little to cause a significant difference $(N=12)$.

For perinatal outcome, the neonatal birth weight was statistically similar in all groups, from 3.31 to $3.37 \mathrm{~kg}(P>0.05$, Fig. 5D). So, the cytoplasmic strings between ICM and mTE cells did not affect the neonatal birth weight.

\section{Discussion}

Nowadays, the main criterion for select blastocyst to transfer is morphological feature, such as the grades for ICM and TE based on Gardner system. However, some essential morphological parameters may be neglected, i.e. cytoplasmic strings among cells. It is reported that some TE cells at the polar-mural junction extend cell projections to the surface of the ICM cells through the cytoplasmic strings by observing the ultrastructure of blastocysts [20]. Salas-Vidal and Lomeli (2004) reported that filamentous 
actin was detected in the cytoplasmic extensions or filopodia in mouse blastocysts [15]. There existed two kinds of filopodia: 1) short filopodia extend both from the ICM and the mural TE into the blastocoel cavity; 2) long, thin filopodia traverse the blastocoel from the mural TE to a central ICM cell. The short filopodia exist abundantly in blastocoel; and about $40 \%$ blastocysts could be observed with the long filopodia.

The cytoplasmic strings between ICM and mTE cells could commonly be observed at blastocyst stage by time lapse system. It is reported that $55-60 \%$ of blastocele could obverse this phenomenon [21]. In present study, even $79.08 \%$ transferred blastocysts could be observed with cytoplasmic strings between ICM and mTE cells. The reason that present percentage is higher in our study may be the cases were transferred blastocysts, rather than all blastocysts. Besides, these blastocysts were selected as the most likely to get pregnancy, so we may conclude the cytoplasmic strings were more visible among good quality blastocysts. Therefore, it is crucial to explore the origin and effect of these strings.

The cytoplasmic strings may be caused due to the immigration from polar TE cells to mural TE [22]. Salas-Vidal and Lomelí (2004) reported that the cytoplasmic strings between ICM and mTE cells may suggest cellular activity [15]. Besides, filopodia are dynamic, appearing to extend and retract in coordination with cell division [21]. The strings were also verified by immunolocalization of the FGFR2 and ErbB3 receptors to indicate a direct communication between the mural TE and the ICM cells [15]. It is also reported that the cytoplasmic string could receive the required mitotic signals from the ICM. Other studies found that filopodia was abundant of E-cadherin, and determined cell elongation behaviors required by compaction. When a-catenin and $\beta$-catenin were knocked down, the filopodia quality was reduced dramatically[21, 23]. So, E-cadherin may be the main ingredient of filopodia. Besides, these cytoplasmic strings not only exist in vitro culture, but also in uteri of volunteers [24]. So, cytoplasmic strings between ICM and mTE cells is a common physiological phenomenon that occurs during cell growth. The presence is conducive to the communication between the mural TE and the ICM cells, conducive to blastocyst development.

In present study, the group of presence of cytoplasmic strings between ICM and mTE cells was more visible with a higher grade of ICM and TE. Therefore, we may conclude that cytoplasmic strings between the mTE and the ICM cells may be an indicator of better quality blastocysts. This may due to the enhanced cellular communication between ICM and mTE cells. Therefore, it is interesting to explore the effect of these strings on the clinical and perinatal outcome of blastocysts.

In order to compare the pregnancy outcomes between blastocysts with present and absent cytoplasmic strings between ICM and mTE cells, clinical pregnancy, live birth and abortion rates were evaluated. A higher clinical pregnancy and live birth rates were observed $(P<0.05)$, but abortion rate was not significantly different $(P>0.05)$. In Thomas Ebner's study, string-positive blastocysts and string-negative blastocysts have similar clinical pregnancy rate $(46.3 \%$ versus $41.2 \%, \mathrm{P}>0.05)$ and live birth rate $(42.7 \%$ versus $30.9 \%, P>0.05)$. Though the live birth rate of string-positive blastocysts is much higher than string-negative blastocysts, no significant difference was observed. This may due to the quantity of 
involved cases, only 82 cycles in string-positive group and 68 cases in string-negative group [16]. If Ebner's study could enlarge the case quantity, a similar conclusion may be summarized. In our study, abortion rate was $10.65 \%$ in present stings group and $12.5 \%$ at absent stings group ( $P>0.05)$. But, among the three groups of string classification, the abortion rate ranged from $8.08-20 \%$. Due to the few cases of abortions, no significant difference was observed $(P>0.05)$. However, this also reminds us to take notice that whether the high abortion was caused by too many strings. For perinatal outcome, the neonatal birth weight was statistically similar in all groups, from 3.31 to $3.37 \mathrm{~kg}(P>0.05)$. So, the cytoplasmic strings between ICM and mTE cells could increase the pregnancy and live birth rates, but did not influence the abortion and birth weight. This may due to the selective and bidirectional communication between mitotic MTE cells and ICM cells induced by cytoplasmic strings. Though the communication between MTE and the ICM could be accomplished by diffusion of secreted molecules into the fluid, this may also bring these signals to cells that have already ceased proliferation. So, cytoplasmic strings between ICM and mTE cells lead to communication more effectively and enable a more efficient delivery of substances and participate in the exploration of the extracellular environment. Therefore, we suspect for the first time that cytoplasmic strings between ICM and mTE cells are a positive phenomenon, which will increase the pregnancy and live birth rates clinically.

\section{Conclusions}

To summarize, these strings were more visible among good quality blastocysts, and caused a higher clinical pregnancy and live birth rates. In conclusion, this study provides the evidence that cytoplasmic strings between ICM and mTE cells would be a positive predictor of pregnancy and live birth outcome in frozen-thawed eSBT cycles. The characterization and functional studies of cytoplasmic strings between ICM and $\mathrm{mTE}$ cells are still required to be investigated.

\section{List Of Abbreviations}

ICM, inner cell mass; mTE, mural trophectoderm; eSBT, elective single blastocyst transfer; IVF, in vitro fertilization; ZP, zona pellucida; KID, known implantation data; COS, controlled ovarian stimulation; HCG, human chorionic gonadotropin; $\mathrm{COC}$, cumulus-oocyte complexe; ICSI, intracytoplasmic sperm injection.

\section{Declarations}

\section{Ethics approval and consent to participate}

Approval was obtained from the ethics committee of Reproductive Medicine Center, Tongji Hospital, Tongji Medical College, Huazhong University of Science and Technology, Hubei, China. The procedures used in this study adhere to the tenets of the Declaration of Helsinki.

\section{Consent for publication}

Not Applicable. 
Availability of data and materials

The datasets used and/or analyzed during the current study are available from the corresponding author on reasonable request.

\section{Competing interests}

The authors declare that they have no conflict of interest.

\section{Funding}

This study was funded by the National Natural Science Foundation of China (81801531 and 82004017$)$. The funders played no role in the writing of the manuscript or the decision to submit it for publication.

\section{Authors' contributions}

B.X. M., B.H. and L. J. made substantial contributions to the design of the study. B.X. M. and B.H. conducted the experiments, analysis and interpretation of data. B.X. M. drafted the work, B.H. and L. J. revised the draft critically for important intellectual content. All authors reviewed and approved the final version of the manuscript.

\section{Acknowledgements}

The authors thank Ms. Fatoumata Touray for her help in modifying the language.

\section{References}

1. Mancuso $\mathrm{A}$ and $\mathrm{J}$ Kapfhamer, With a good quality blastocyst, single embryo transfer remains the best choice. Fertil Steril 2018; 110(4): 631. DOI: 10.1016/j.fertnstert.2018.06.024.

2. Du QY, EY Wang, Y Huang, XY Guo, YJ Xiong, YP Yu, et al., Blastocoele expansion degree predicts live birth after single blastocyst transfer for fresh and vitrified/warmed single blastocyst transfer cycles. Fertil Steril 2016; 105(4): 910-919 e1. DOI: 10.1016/j.fertnstert.2015.12.014.

3. Lan KC, YJ Tseng, YR Su, TY Lin, and YC Lin, Evaluation of the effect of the elective blastocyst-stage embryo transfer and freezing strategy on the abandonment of frozen embryos under the Taiwan National Assisted Reproduction Act. J Assist Reprod Genet 2020; 37(4): 973-982. DOI: 10.1007/s10815-020-01699-5.

4. Sutherland K, J Leitch, H Lyall, and BJ Woodward, Time-lapse imaging of inner cell mass splitting with monochorionic triamniotic triplets after elective single embryo transfer: a case report. Reprod Biomed Online 2019; 38(4): 491-496. DOI: 10.1016/j.rbmo.2018.12.017.

5. Glujovsky D and C Farquhar, Cleavage-stage or blastocyst transfer: what are the benefits and harms? Fertil Steril 2016; 106(2): 244-50. DOI: 10.1016/j.fertnstert.2016.06.029. 
6. Reignier A, JM Girard, J Lammers, S Chtourou, T Lefebvre, P Barriere, et al., Performance of Day 5 KIDScore morphokinetic prediction models of implantation and live birth after single blastocyst transfer. J Assist Reprod Genet 2019; 36(11): 2279-2285. DOI: 10.1007/s10815-019-01567-x.

7. Devine K, MT Connell, KS Richter, CI Ramirez, ED Levens, AH DeCherney, et al., Single vitrified blastocyst transfer maximizes liveborn children per embryo while minimizing preterm birth. Fertil Steril 2015; 103(6): 1454-60 e1. DOI: 10.1016/j.fertnstert.2015.02.032.

8. Evans J, NJ Hannan, TA Edgell, BJ Vollenhoven, PJ Lutjen, T Osianlis, et al., Fresh versus frozen embryo transfer: backing clinical decisions with scientific and clinical evidence. Hum Reprod Update 2014; 20(6): 808-21. DOI: 10.1093/humupd/dmu027.

9. Papanikolaou EG, M Camus, EM Kolibianakis, L Van Landuyt, A Van Steirteghem, and P Devroey, In vitro fertilization with single blastocyst-stage versus single cleavage-stage embryos. N Engl J Med 2006; 354(11): 1139-46. DOI: 10.1056/NEJMoa053524.

10. Zeng MF and LM Li, Frozen blastocyst transfer reduces incidence of ectopic pregnancy compared with fresh blastocyst transfer: a meta-analysis. Gynecol Endocrinol 2019; 35(2): 93-99. DOI: 10.1080/09513590.2018.1497154.

11. Wei D, JY Liu, Y Sun, Y Shi, B Zhang, JQ Liu, et al., Frozen versus fresh single blastocyst transfer in ovulatory women: a multicentre, randomised controlled trial. Lancet 2019; 393(10178): 1310-1318. DOI: $10.1016 /$ S0140-6736(18)32843-5.

12. Cutting R, Single embryo transfer for all. Best Pract Res Clin Obstet Gynaecol 2018; 53: 30-37. DOI: 10.1016/j.bpobgyn.2018.07.001.

13. Gardner DK and WB Schoolcraft, Culture and transfer of human blastocysts. Curr Opin Obstet Gynecol 1999; 11(3): 307-11. DOI: 10.1097/00001703-199906000-00013.

14. Hardarson T, L Van Landuyt, and G Jones, The blastocyst. Hum Reprod 2012; 27 Suppl 1: i72-91. DOI: 10.1093/humrep/des230.

15. Salas-Vidal E and H Lomeli, Imaging filopodia dynamics in the mouse blastocyst. Dev Biol 2004; 265(1): 75-89. DOI: 10.1016/j.ydbio.2003.09.012.

16. Ebner T, O Sesli, S Kresic, S Enengl, B Stoiber, E Reiter, et al., Time-lapse imaging of cytoplasmic strings at the blastocyst stage suggests their association with spontaneous blastocoel collapse. Reprod Biomed Online 2020; 40(2): 191-199. DOI: 10.1016/j.rbmo.2019.11.004.

17. Huang B, D Hu, K Qian, J Ai, Y Li, L Jin, et al., Is frozen embryo transfer cycle associated with a significantly lower incidence of ectopic pregnancy? An analysis of more than 30,000 cycles. Fertil Steril 2014; 102(5): 1345-9. DOI: 10.1016/j.fertnstert.2014.07.1245.

18. Jin L, M Wang, J Yue, GJ Zhu, and B Zhang, Association between TSH Level and Pregnancy Outcomes in Euthyroid Women Undergoing IVF/ICSI: A Retrospective Study and Meta-analysis. Curr Med Sci 2019; 39(4): 631-637. DOI: 10.1007/s11596-019-2084-5.

19. Huang $B, X$ Ren, $L$ Zhu, L Wu, $H$ Tan, N Guo, et al., Is differences in embryo morphokinetic development significantly associated with human embryo sex?dagger. Biol Reprod 2019; 100(3): 618-623. DOI: 10.1093/biolre/ioy229. 
20. Ducibella T, DF Albertini, E Anderson, and JD Biggers, The preimplantation mammalian embryo: characterization of intercellular junctions and their appearance during development. Dev Biol 1975; 45(2): 231-50. DOI: 10.1016/0012-1606(75)90063-9.

21. Coticchio G, C Lagalla, R Sturmey, F Pennetta, and A Borini, The enigmatic morula: mechanisms of development, cell fate determination, self-correction and implications for ART. Hum Reprod Update 2019; 25(4): 422-438. DOI: 10.1093/humupd/dmz008.

22. Scott LA, Oocyte and embryo polarity. Semin Reprod Med 2000; 18(2): 171-83. DOI: 10.1055/s-200012556.

23. Fierro-Gonzalez JC, MD White, JC Silva, and N Plachta, Cadherin-dependent filopodia control preimplantation embryo compaction. Nat Cell Biol 2013; 15(12): 1424-33. DOI: 10.1038/ncb2875.

24. Munne S, ST Nakajima, S Najmabadi, MV Sauer, MJ Angle, JL Rivas, et al., First PGT-A using human in vivo blastocysts recovered by uterine lavage: comparison with matched IVF embryo controlsdagger. Hum Reprod 2020; 35(1): 70-80. DOI: 10.1093/humrep/dez242.

\section{Figures}




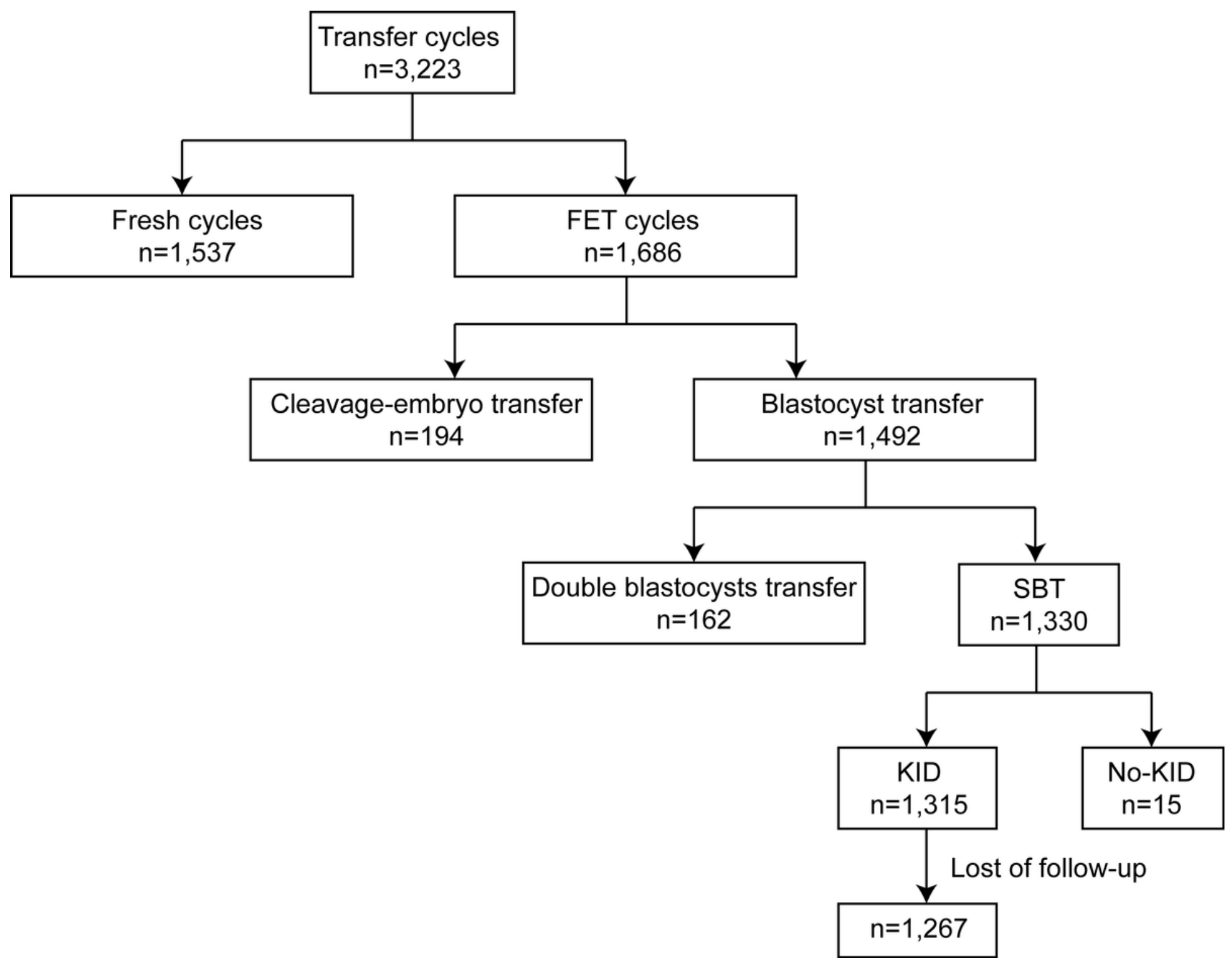

Figure 1

Schematic presentation of the study design. SBT, single blastocyst transfer; KID, known implantation data. FET, frozen embryo transfer. 


\section{Class I}
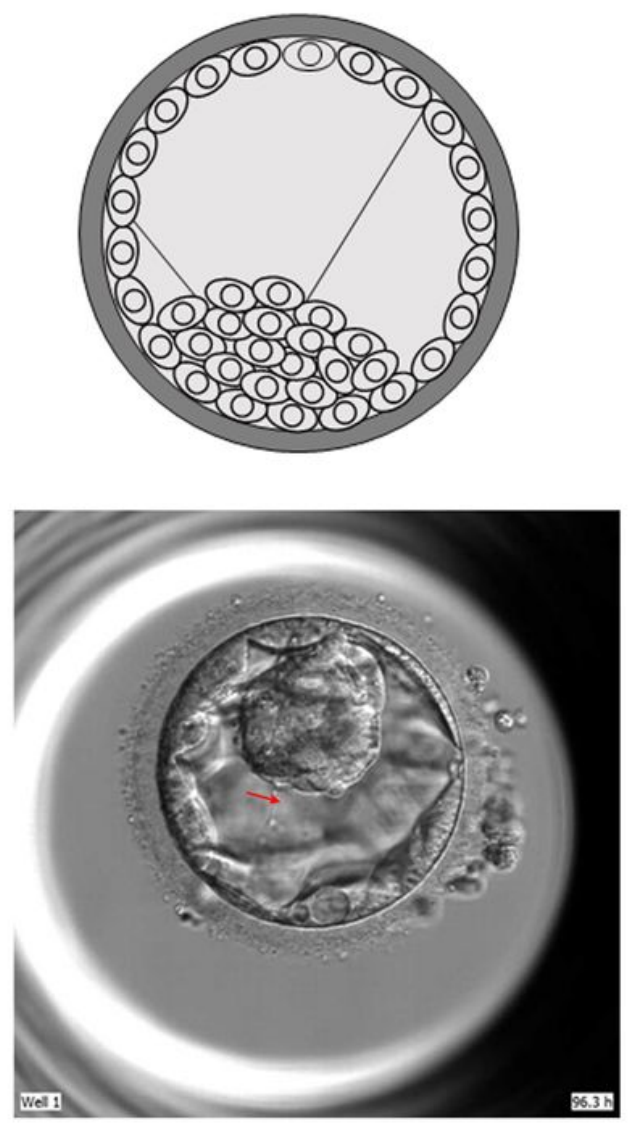

\section{Class II}
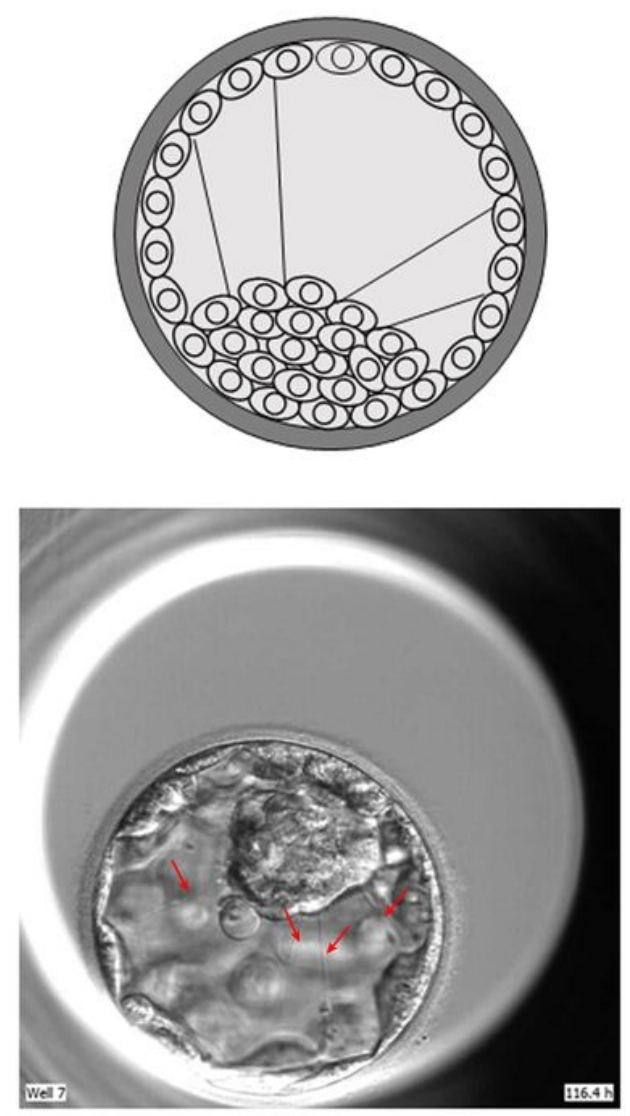

\section{Class III}
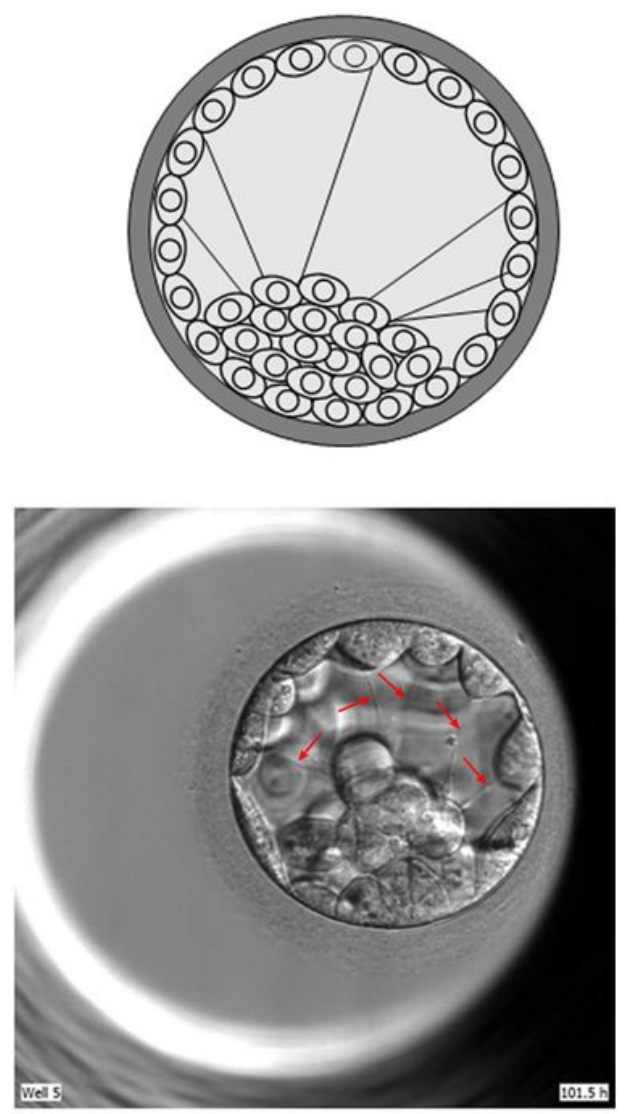

Figure 2

Classification of cytoplasmic strings between ICM and mTE cells. Threads were classified into three groups, based on their quantity: single and double string(s) (I), three to four strings (II) and more than four strings (III). ICM, inner cell mass; mTE, mural trophectoderm. 
A

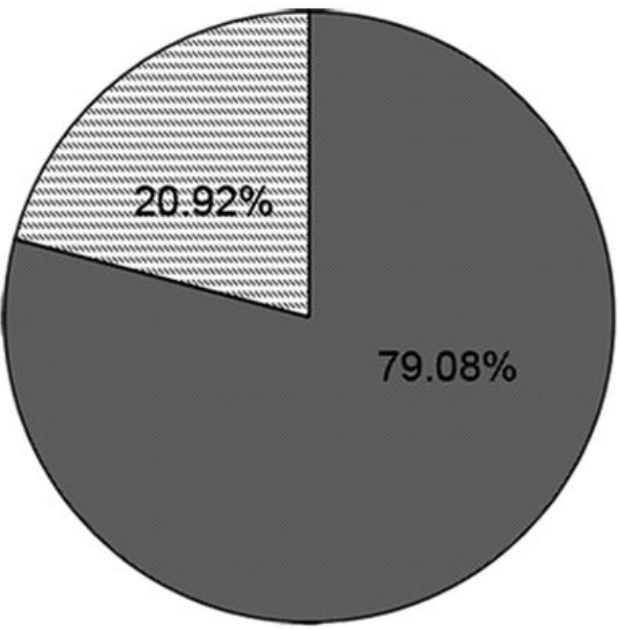

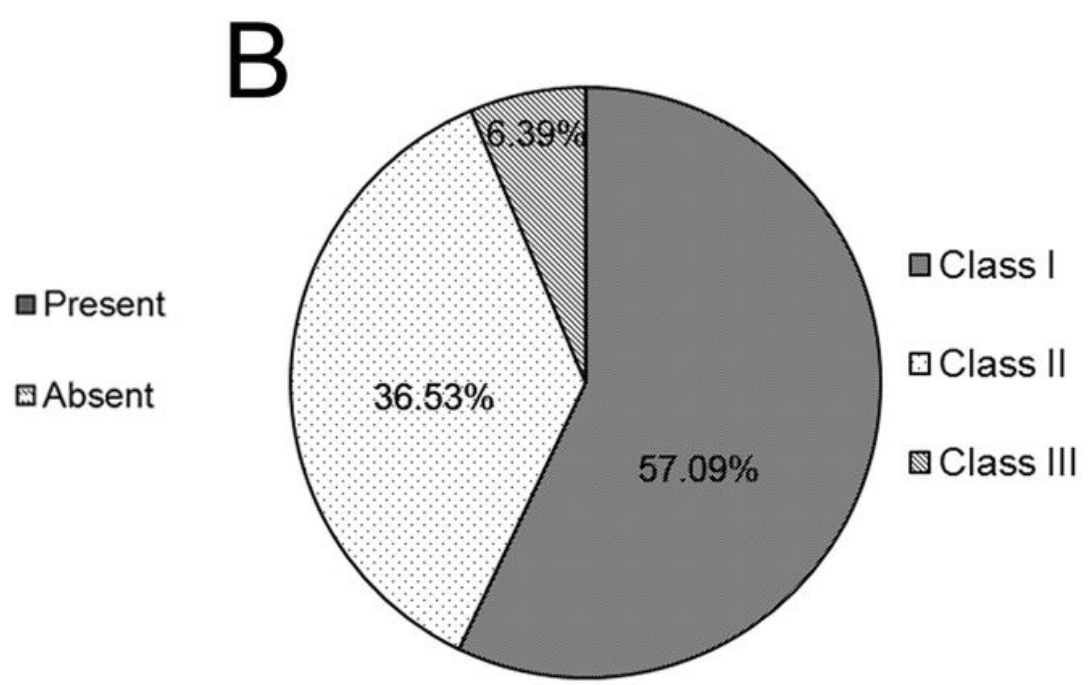

Figure 3

The quantity and percentage of different groups. (A) Present and Absent group of cytoplasmic strings between ICM and mTE cells; (B) different classifications of present strings group. ICM, inner cell mass; mTE, mural trophectoderm. 

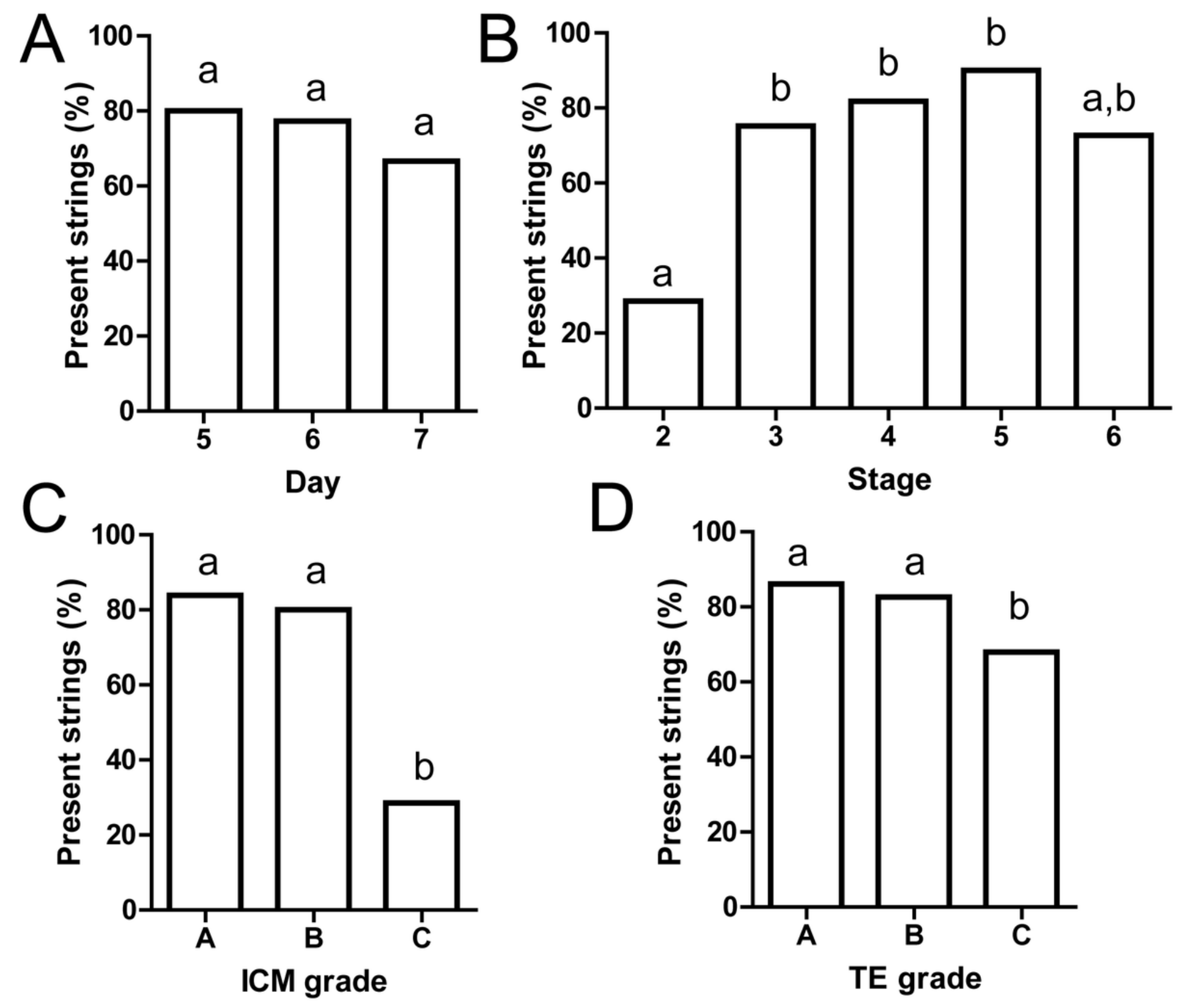

Figure 4

The present frequency of cytoplasmic strings between ICM and MTE cells. (A) different blastocyst ages; (B) different blastocyst stages; (C) different ICM grades; (D) different TE grades. The same superscript letter is not significantly different at $\mathrm{P}<0.05$ level. ICM, inner cell mass; $\mathrm{mTE}$, mural trophectoderm. 

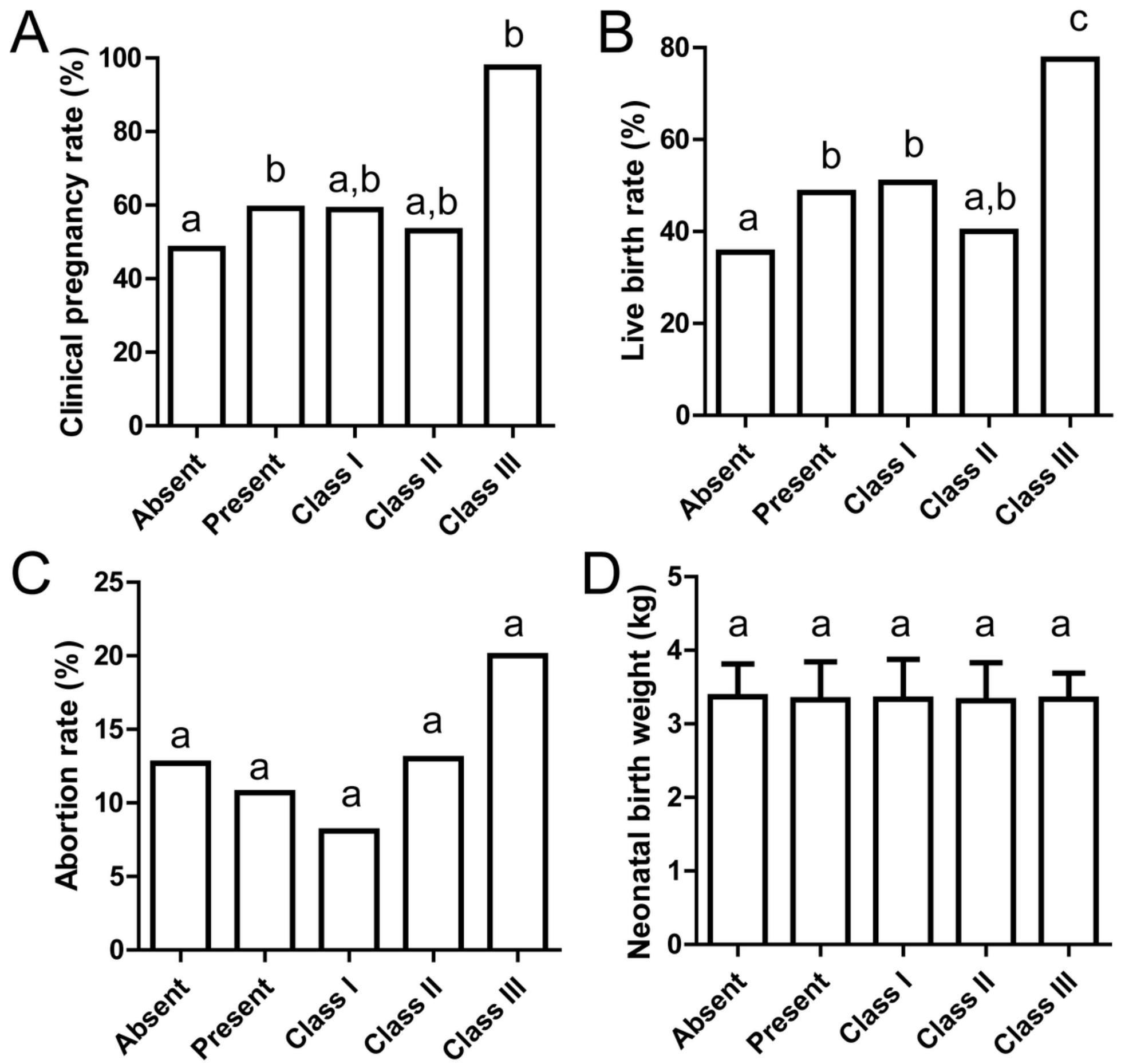

Figure 5

The outcomes of blastocyst with cytoplasmic strings between ICM and mTE cells. (A) clinical pregnancy rate; (B) live birth rate; (C) abortion rate; (D) neonatal birth weight. The same superscript letter is not significantly different at $\mathrm{P}<0.05$ level. ICM, inner cell mass; $\mathrm{mTE}$, mural trophectoderm. 